\title{
Consensus may not be systematic
}

\section{Jim Bader and Dan Shugars \\ Department of Operative Dentistry, University of North Carolina, Chapel Hill, North Carolina, USA}

Evidence-Based Dentistry (2002) 3, 91-92. doi:10.1038/sj.ebd.6400105

Consensus statements are designed to answer a series of four to six questions concerning efficacy, risk and clinical applications, and to recommend directions for future research (see the National Institutes of Health Office of Medical Applications of Research website, www.consensus.nih.gov/about/ about.htm). ${ }^{1}$ Consensus statements are written by broad-based, independent panels of non-government, nonadvocate individuals who are knowledgeable in the field of medical science that is under consideration. Panels typically include investigators, healthcare providers, methodologists and a public representative. Following a dayand-a-half of scientific presentations and public testimony, the panel convenes in an executive session to write the draft consensus statement. On the third and final day of the conference, the statement is circulated to the conference audience for comment. Afterwards, the panel resolves any conflicting recommendations and releases a revised statement at the end of the conference. The conference on dental caries was the first Consensus Development Conference (CDC) to be based on a series of systematic reviews of the relevant literature. ${ }^{2}$ A majority, but not all, of the principal presentations during the conference described the results of such reviews. Complete reports including detailed evidence tables were made available to the panel in advance of the conference. Presentations consisted of sets of short, 10-20 min summaries of these reports followed by questions to groups of presenters by the panel and the audience.

The systematic reviews appearing in this issue addressed aspects of two of the questions dealt with by the CDC. The first of these questions was, "What are the best methods available for the primary prevention of dental caries initiation throughout life?". Our review for this question was limited to professionally applied primary prevention in individuals deemed to be at high risk of developing new lesions. ${ }^{3}$ We reported that there was fair evidence for the effectiveness of fluoride varnishes for this application, and that the strength of the evidence for effectiveness of all other interventions was insufficient. We indicated that we found the evidence to be suggestive of effectiveness for chlorhexidine gels and varnishes, for combined chlorhexidine and fluoride interventions and for sucrose-free gum.

The second question was, "What are the best treatments available for reversing or arresting the progression of early dental caries?". Here our review rated the available evidence as insufficient for all professionally applied methods. ${ }^{3}$

The original consensus statement, presented for discussion in the morning of the third day of the conference, repeated our assessment of fair evidence of effectiveness only for fluoride varnishes for primary prevention in high-risk subjects. It was suggested that additional studies were needed to identify the best interventions for these individuals. In contrast, however, the statement did not reflect the conclusions of the systematic review with respect to arresting and reversing noncavitated lesions. The consensus statement noted that clinical strategies for reversal already existed and were similar to those for primary prevention. The statement went on to indicate that the quantity and quality of data varied by treatment, but that data supported the use of fluoride varnishes. For fluoride rinse and gel applications, the evidence was suggestive but not definitive. The statement also indicated that the data for chlorhexidine varnishes and gels were promising. In addition, data for combinations of chlorhexidine, fluoride and/or sealants were noted to be suggestive of efficacy. In essence, the consensus statement applied our assessment of the evidence for the effectiveness of methods for the primary prevention of lesions in highrisk individuals to the question concerning the effectiveness of methods for arresting and reversing early carious lesions.

We noted the apparent error in the morning comment session. The revised consensus statement, issued later that day, remained unchanged. We then sent a memorandum, again voicing our concern, to the chairman of the consensus panel and to the Director of the Office of Medical Application of Research. A final revised version of the consensus statement appeared one week later with two changes directed towards our concern. ${ }^{4}$ First, the statement about the existence of clinical strategies was softened to say that they 
may exist. Second, a sentence was added to the statement noting that, "the panel believes that existing strategies for primary prevention in the general population, as well as preventive strategies demonstrated to be effective in high-risk individuals, are also likely to be effective in arresting or reversing early lesions."

The problem remains that the consensus statement misrepresents the scientific literature on professionally applied methods for arresting and reversing early carious lesions. The systematic review was quite explicit in noting that there is almost no evidence in the literature addressing this issue. This finding was not questioned during the conference. Yet the revised consensus statement suggests that there is sufficient evidence to demonstrate, or at least to suggest, the effectiveness of a number of professionally applied interventions in arresting or reversing early dental caries. In fact, this evidence does not exist. Although the consensus panel stated that it believes the results of primary prevention studies are applicable to the question concerning arrest and reversal, it offered no support for that belief. Further, the panel offered no explanation for why the results of our review of effectiveness in high-risk individuals were applied almost verbatim to this question.

Clearly the non-surgical arrest and reversal of early carious lesions is an important clinical topic, and clinicians should know about the evidence for this action. One of the great promises of systematic reviews is that they will lead to more evidence-based treatment. $^{5}$ Yet the appropriate use of systematic reviews in clinical practice depends on additional steps to 'preprocess' the evidence, which Guyatt and colleagues identified as synopses and information systems. ${ }^{6}$ The consensus statement is a synopsis, one that will undoubtedly be incorporated into future information systems such as texts, practice guidelines and clinical pathways. Such synopses need to summarise the evidence accurately. It is unfortunate that, with respect to arrest and reversal of early carious lesions, the consensus statement does not succeed. The damage is not irreparable, and the consensus panel's belief may well be supported when all the evidence is finally available. Regardless, in this instance the consensus precedes the evidence.

1. National Institutes of Health, Office of Medical Applications of Research. www.consensus.nih.gov/about/about.htm

2. National Institutes for Health. National Institutes for Health Consensus

Development Conference: Diagnosis \& Management of Dental Caries Throughout Life. Bethesda: Office of the Director, National Institutes for Health; 2001 (March 26) [program and abstracts].

3. Bader J, Shugars S, Bonito A. A systematic review of selected caries prevention and management methods. Community Dent Oral Epidemiol 2001; 29:399-401.

4. NIH. Consensus Development Conference Statement: diagnosis and management of dental caries throughout life. http:// consensus.nih.gov/cons/115/ 115_statement.htm

5. Mulrow C, Cook D (Editors). Systematic Reviews, Synthesis of Best Evidence for Health Care Decisions Philadelphia: American College of Physicians; 1998.

6. Guyatt $\mathrm{GH}$, Haynes RB, Jaeschke RZ, Cook DJ, Green L, Naylor CD, Wilson MC, Richardson WS. Users' guides to the medical literature. XXV. Evidence-based medicine: principles for applying the users' guides to patient care. JAMA 2000; 284:1290-1296. 